Review Article

\title{
From yeast to humans: Understanding the biology of DNA Damage Response (DDR) kinases
}

\author{
José Renato Rosa Cussiol ${ }^{1}$ (D), Bárbara Luísa Soares ${ }^{2}$ and Francisco Meirelles Bastos de Oliveira ${ }^{2}$ (iD \\ ${ }^{1}$ Departamento de Bioquímica, Universidade Federal de São Paulo, São Paulo, SP, Brazil. \\ ${ }^{2}$ Instituto de Biofísica Carlos Chagas Filho, Universidade Federal do Rio de Janeiro, Rio de Janeiro, RJ, \\ Brazil.
}

\begin{abstract}
The DNA Damage Response (DDR) is a complex network of biological processes that protect cells from accumulating aberrant DNA structures, thereby maintaining genomic stability and, as a consequence, preventing the development of cancer and other diseases. The DDR pathway is coordinated by a signaling cascade mediated by the PI3K-like kinases (PIKK) ATM and ATR and by their downstream kinases CHK2 and CHK1, respectively. Together, these kinases regulate several aspects of the cellular program in response to genomic stress. Much of our understanding of these kinases came from studies performed in the 1990s using yeast as a model organism. The purpose of this review is to present a historical perspective on the discovery of the DDR kinases in yeast and the importance of this model for the identification and functional understanding of their mammalian orthologues.
\end{abstract}

Keywords: Yeast, genome instability, DNA damage response, cell cycle checkpoint, kinase.

Received: March 7, 2019; Accepted: July 19, 2019.

\section{Introduction}

Despite its apparent stability, DNA can undergo significant changes in its structure. Spontaneous hydrolysis, oxidation and non-enzymatic methylation of DNA nitrogen bases can induce tens of thousands of lesions per day (Lindahl and Nyberg, 1972; Lindahl, 1993). In addition, environmental agents such as genotoxic chemicals, ultraviolet light (UV) and ionizing radiation (IR) can increase the frequency of single strand breaks (SSBs) and double strand breaks (DSBs) (Friedberg, 2008; Giglia-Mari et al., 2011). Replicating cells are particularly susceptible to DNA lesions because the progression of replication forks can be hampered by DNA adducts, DNA-RNA hybrids, proteinDNA complexes or depletion of dNTP pools (Lambert and Carr, 2013). These lesions, if left unrepaired, can lead to genomic instability, which is a hallmark of cancer and other diseases. Thus, eukaryotic cells regulate a set of biological processes collectively entitled as DNA Damage Response (DDR) (Ciccia and Elledge, 2010).

The DDR comprises multiple DNA repair and DNA damage tolerance pathways, as well as cell cycle checkpoints. Therefore, the existence of a DNA damage signaling pathway responsible for ensuring efficient, accurate

Send correspondence to Francisco Meirelles Bastos de Oliveira. Instituto de Biofísica Carlos Chagas Filho, Universidade Federal do Rio de Janeiro (UFRJ), Av. Carlos Chagas Filho 373, Cidade Universitária, 21941-902 Rio de Janeiro, RJ, Brazil. E-mail: francisco@ biof.ufrj.br. and timely DDR is imperative for cell survival. One of the most important layers of DDR regulation comprises a complex signaling network mediated by serine/threonine kinases members of the phosphatidylinositol-3-kinase-like kinase family (PI3K-like or PIKKs). In mammals, this signaling network is orchestrated by the DDR kinases ATR, ATM and DNA-PK (Blackford and Jackson, 2017). These kinases act as DNA damage sensors and effectors, recognizing alterations in the DNA molecule and eliciting a signaling cascade through the phosphorylation of hundreds of proteins (Falck et al., 2005; Marechal and Zou, 2013).

Since the discovery of the DDR kinases, much progress has been made in the understanding of their role in genome stability. Due to its biological relevance, DDR is highly conserved from yeast to humans (Table 1). Of note, the identification and dissection of the molecular function of the DDR kinases was only possible because of the contribution of several laboratories studying DNA damage signaling in model organisms such as Saccharomyces cerevisiae and Schizosaccharomyces pombe. Therefore, yeast has been placed as an attractive model to uncover the molecular mechanisms behind the function of the DDR kinases. In this review, we offer a historical perspective of the identification and characterization of DDR kinases by following the chronology of classical studies and highlighting their contributions to the understanding of the DDR pathway. 
Table 1 - DDR kinases homologs in yeast and human.

\begin{tabular}{lcc}
\hline S. cerevisiae & S. pombe & Human \\
\hline MEC1 & rad3 & ATR \\
TEL1 & tel1 $*$ & ATM \\
RAD53 & $c d s 1$ & CHK2 \\
CHK1 & $c h k 1$ & CHK1 \\
DUN1 & - & - \\
- & - & DNA-PK \\
\hline
\end{tabular}

* (Naito et al., 1998)

\section{Dun1, the first kinase associated with DDR in eukaryotes}

In the late $1980 \mathrm{~s}$, in an attempt to identify a recombinase in $S$. cerevisiae, Stephen Elledge accidentally isolated the gene encoding a subunit of ribonucleotide reductase (RNR2) (Elledge and Davis, 1987; Elledge, 2015). The initial disappointment, however, turned into curiosity when $R N R 2$ expression was shown to be dependent on treatment with drugs that interfere with DNA replication (Elledge and Davis, 1987, 1989). This result suggested that eukaryotic cells could modulate nucleotide synthesis in response to DNA damage caused during replication arrest. Reinforcing this hypothesis, in the following years, genes encoding other subunits of the ribonucleotide reductase such as RNR1 and RNR3 were isolated, both presenting expression patterns similar to those observed for $R N R 2$ (Elledge and Davis, 1990). In the early 90s, in order to understand the molecular basis of this signaling mechanism, the Elledge laboratory developed a genetic screen to identify genes involved in the regulation of $R N R 3$ expression. The approach aimed to identify mutants of $S$. cerevisiae that repressed $R N R 3$ expression upon treatment with hydroxyurea (HU), a DNA synthesis inhibitor. Mutants isolated in this screen were referred to as DNA-damage uninducible (dun) (Zhou and Elledge, 1993). Among the isolated candidates the most promising was a serine/threonine protein kinase named Dun1 (Figure 1). The sensitivity of dun 1 mutants to HU suggested that upregulation of ribonucleotide reductase was an important event for cell tolerance to DNA replication arrest. Most importantly, this observation reinforced the existence of a signaling pathway responding to DNA damage in eukaryotic cells. Indeed, metabolic labeling with ${ }^{32} \mathrm{P}$-labeled phosphate showed that Dun1 became highly auto-phosphorylated in response to $\mathrm{HU}$, suggesting that its function was actively modulated during DDR (Zhou and Elledge, 1993). In addition to autophosphorylation, Dun1 presented another phosphorylated form that occurred independently of its catalytic activity. Although its function was not clear, this raised the possibility that upstream kinases might be involved in regulating this signaling pathway (Figure 2). Curiously, although dun 1 mutants showed reduced expression of ribonucleotide reductase, the cell cycle checkpoints remained intact (Zhou and Elledge, 1993). This suggested a possible ramification of the DDR in $S$. cerevisiae where, in addition to Dun1, other signaling components were required to regulate different functions necessary to protect cells against damage arising during DNA replication (Figure 2).

\section{Mec1, a yeast PI3K-like kinase linking cell cycle checkpoints and meiotic recombination}

In the early 90s, Lee Hartwell and Ted Weinert observed that the combination of mitotic checkpoint mutant rad9 (radiation sensitive 9) with $c d c 13$ (cell division cycle $\underline{13}$ ), showed a striking loss of viability when compared to single mutants alone (Weinert and Hartwell, 1993). cdc13 was defective for telomere metabolism, accumulating aberrant DNA structures near the end of the chromosomes. The authors inferred that loss of viability of the double mutant could be attributed to cell division with aberrant DNA structures. Based on the genetic interaction observed for cdc13 rad9, Lee Hartwell and Ted Weinert developed a screen to identify new genes involved in the regulation of the mitotic checkpoint. By inducing random mutations in a $c d c 13$ mutant and analyzing more than 12,000 strains, the authors identified four mutants with a strong negative genetic interaction. These mutants were named mitosis entry checkpoint (mec) and included mec1, mec2, mec 3 and rad9 itself (Weinert et al., 1994) (Figure 1). Less than one year

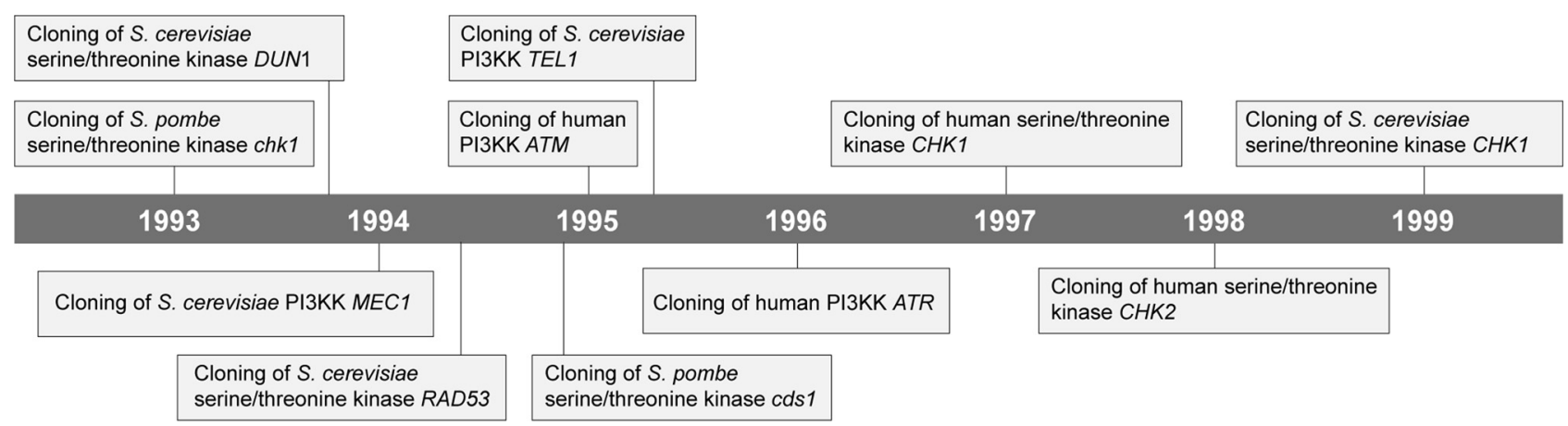

Figure 1 - Timeline for the discovery of DDR kinases in yeast and human. Although the gene encoding S. pombe Chk1 was identified before Dun1, its kinase activity was associated with DDR only in 1996 (Walworth and Bernards, 1996). 


\section{S. cerevisiae}

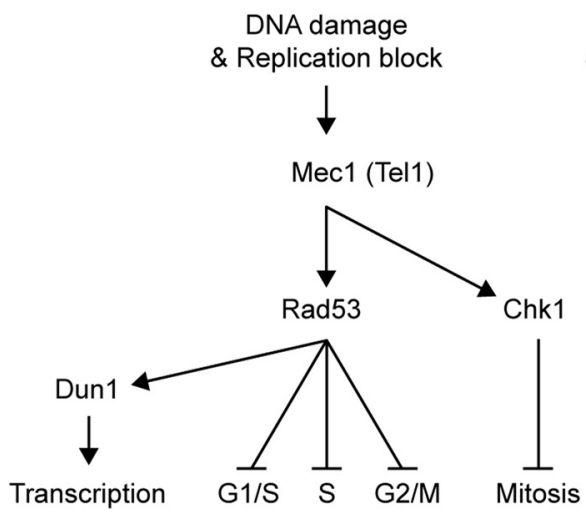

S. pombe

DNA damage

\& Replication block

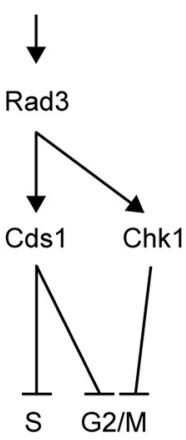

Human

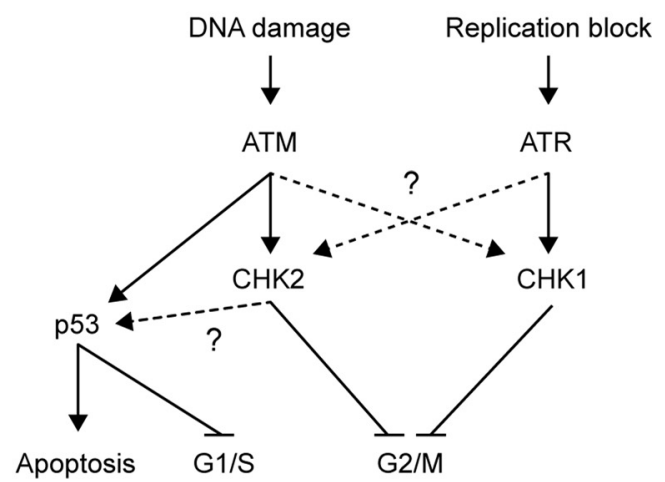

Figure 2 - Schematic representation of the signaling network of DDR kinases in yeast and human. In S. cerevisiae, DNA damage and replication block signal is preferentially transduced from Mec1 to Rad53 and Chk1 with Tel1 showing an overlap with Mec1. Rad53 inhibits the G1/S, S phase and G2/M cell cycle transitions and activates transcription in a Dun1-dependent manner. Chk1 acts in parallel to Rad53 inhibiting mitosis by preventing anaphase entry. In S. pombe, DNA damage and replication block signal is preferentially transduced from Rad3 to Cds1 and Chk1. Cds1 inhibits S phase and reinforces G2/M inhibition together with Chk1. In mammalian cells, ATM signals to $\mathrm{p} 53$, which in turn activates apoptosis and inhibits G1/S cell cycle transition (Kastan et al., 1991; Lowe et al., 1993). While ATM signals to CHK2, ATR signals to CHK1 in response to DNA replication inhibition. Both CHK1 and CHK2 inhibit the G2/M cell cycle transition, although at that time their roles during S phase progression were unknown. Also, there was still no evidence on the crosstalk between ATM/ATR with CHK1/CHK2, nor between CHK2 and p53. Dashed lines and interrogation marks represent unknown pathways at that time.

later, mec 1 was also identified as sad 3 and esr 1 by two independent research groups. In the first case, sad3 was identified by Stephen Elledge's group in a screen performed to identify HU-sensitive mutants. These mutants were named

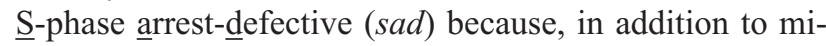
totic checkpoint defects, they were also defective in the $\mathrm{S}$ phase checkpoint (Allen et al., 1994). Reinforcing this finding, Paulovich and Hartwell (1995) demonstrated that slowing of replication forks during DNA damage is an active process dependent on $M E C 1$.

Based on the hypothesis that meiosis II was similar to a mitotic division, Ryuichi Kato and Hideyuki Ogawa performed a screen to identify mutants that were not only sensitive to DNA damage agents but also defective in meiotic recombination. Using this approach the authors identified and cloned an essential gene required for DNA repair and meiotic recombination named ESRI (Kato and Ogawa, 1994). Interestingly, the ESRI-encoded protein showed high similarity with phosphatidylinositol 3-kinases (PI3K). However, at that time it was unclear whether Esr1-mediated signal transduction was restricted to lipid phosphorylation.

As described in the following sections, the discovery of other kinases associated with the DDR reinforced functional and structural divergences between Esr1 and classical PI3Ks (Keith and Schreiber, 1995). For this reason, together with Esr1, these kinases were then referred to as PI3K-like protein kinase (PIKKs) For the purpose of this review and following the chronology of identification, henceforth, MEC1/ESR1/SAD3 will be referred to as MEC1.

\section{Tel1, the yeast ortholog of ATM, suggests the existence of parallel pathways in the DDR}

Ataxia telangiectasia (A-T) is an autosomal recessive syndrome characterized by neurodegeneration, immunodeficiency and cancer predisposition. Cells derived from A-T patients show genomic instability and are highly sensitive to IR (Shiloh and Rotman, 1996). In the early 1990s, the main hypothesis for this phenotype suggested a dysfunctional cell cycle checkpoint (Beamish et al., 1994). Over more than five years, the extensive work of several research groups helped to narrow down the genomic region containing the defective gene potentially associated with the A-T phenotype (Gatti et al., 1988; McConville et al., 1994; Rotman et al., 1994; Lange et al., 1995). Finally, in 1995, a consortium lead by Yosef Shiloh's laboratory cloned the $A T M$ (ataxia telangiectasia mutated) gene and identified its respective mutations in A-T patients (Savitsky et al., 1995a,b) (Figure 1).

The amino acid sequence encoded by ATM showed similarity with the PIKK Mec1 cloned a few months earlier in S. cerevisiae (Keith and Schreiber, 1995; Savitsky et al., 1995a). In addition to Mec1, ATM also showed strong similarity to the amino acid sequence encoded by the $S$. cerevisiae open reading frame (ORF) YBL088 (Savitsky et al., 1995b). At that time, two research groups independently identified and cloned the gene correspondent to YBL088 (Greenwell et al., 1995; Morrow et al., 1995). Interested in understanding the mechanisms that led to telomere maintenance defects in the tel1 mutant, Greenwell et al. (1995) cloned a DNA fragment containing TEL1, and interestingly, the analysis of the amino acid sequence encoded by TEL1 was identical to the product of YBL088 (Figure 1). At 
the same time, surprised by the enormous similarity between the amino acids sequences encoded by $A T M$ and YBL088, Morrow et al. (1995) cloned the gene correspondent to YBL088 and, aware of the parallel work of Greenwell, referred to the gene also as TEL1 (Figure 1). Corroborating the functional conservation between ATM and TEL1, tell mutants showed an increase in the frequency of mitotic recombination and loss of chromosomes similar to that observed in A-T cells (Greenwell et al., 1995). However, tell mutants showed no sensitivity to genotoxic agents, suggesting the existence of parallel pathways that could bypass Tel1 function upon DNA damage conditions (Greenwell et al., 1995). Indeed, the similarities between the primary structures of Mec1 and Tell suggested a functional overlap between these two proteins (Morrow et al., 1995) (Figure 2). The authors confirmed this, in part, by showing that an extra copy of TEL1 was able to partially rescue the sensitivity in Mec1-deficient cells treated with IR, UV or HU (Morrow et al., 1995). Interestingly, although mecl mutants were more sensitive to genotoxic agents than tell, they did not display deficiencies in telomere maintenance (Greenwell et al., 1995; Morrow et al., 1995). This suggested that despite the functional overlap, these kinases could also have functions dependent on the type of DNA damage: Mec1 would be preferentially related to the response to DNA damage induced by IR, UV and HU while Tell would be involved in the response associated with damaged telomeres. Although Mec1 and ATM dysfunctions were phenotypically similar, ATM had greater similarity to Tel1. Therefore, it was plausible to infer the existence of a $M E C 1$ ortholog capable of exerting parallel functions to $A T M$ in human cells (Figure 2).

\section{ATR, the human ortholog of Mec1, has a role in the response to DNA damage caused during DNA replication}

rad3 of Schizosaccharomyces pombe was previously identified as a radiation sensitive mutant with defects in the cell cycle checkpoints (al-Khodairy and Carr, 1992; Jimenez et al., 1992). A few years after the characterization of rad3, Savitsky et al. (1995a) demonstrated a high similarity between ATM, Mec1 and a partial sequence of Rad3. Motivated by this similarity, Tony Carr's laboratory isolated the coding region corresponding to $\mathrm{Rad} 3$ and demonstrated the presence of C-terminus consensus sequences that defined Rad3 as a new member of the PIKK family (Bentley et al., 1996) (Figure 1). They also demonstrated that a kinasedead mutant of Rad3 recapitulated the phenotypes described for its null mutant, suggesting that kinase activity was essential for $\operatorname{Rad} 3$ function. In addition, $\operatorname{Rad} 3$ immunoprecipitates were shown to exhibit an associated protein kinase activity, supporting the ability of PIKK to catalyze the phosphorylation of protein substrates (Bentley et al., 1996).

Although belonging to the same family of PIKK, sequence analysis of Rad3, Mec1, Tel1 and ATM suggested an evolutionary divergence in two distinct subfamilies. One subfamily comprised of $\operatorname{Rad} 3 / \mathrm{Mec} 1$ and the other of Tel1/ATM (Table 1). However, unlike the human ATM, which is closely related to TEL1, the existence of a human ortholog for $\mathrm{MECl}$ and rad3 remained unknown. In an attempt to identify the human ortholog of rad3, Tony Carr's laboratory used degenerated PCR based on the sequences of $\operatorname{Rad} 3$ and $\mathrm{Mec} 1$ and subsequently screened a cDNA library isolating the coding region of ATR (ataxia telangiectasia and rad3-related) (Bentley et al., 1996) (Figure 1). At the same time, Cimprich et al. (1996) relied on an expressed sequence tag (EST) with sequence similarity to the PI3K-related kinases FRAP, Tor $1 p$ and Tor $2 p$ to isolate the cDNA corresponding to FRP1 (FRAP-related protein $\underline{1}$ ). $F R P 1$ sequence was shown to be identical to that of $A T R$ and, by convention, was referred to by the same name (Figure 1).

ATR presented elements that characterized it as a PIKK showing higher similarity to $\operatorname{Rad} 3 / \mathrm{Mec} 1$ than to ATM/Tel1 (Keith and Schreiber, 1995; Bentley et al., 1996). In addition, the overexpression of ATR was able to rescue the sensitivity of a mecl partial defective mutant, demonstrating a functional conservation between these kinases (Bentley et al., 1996). These observations reinforced the idea that $A T R$ was the human ortholog of $M E C 1 / \mathrm{rad} 3$ while $A T M$ was the ortholog of TEL1 (Table 1 and Figure 2).

Previous comparative studies between Mec1 and Tell suggested a possible ramification of the DDR pathway, where the different kinases would respond to different types of DNA insults (Morrow et al., 1995). Identification of ATR and ATM suggested that, as in yeast, these kinases would also perform specialized functions in mammalian cells. However, due to the absence of an available model to mimic ATR defects, functional studies on this kinase were only possible in 1998, when Cliby et al. (1998) developed a dominant negative mutant based on the overexpression of a kinase-dead allele of ATR. These experiments confirmed that, unlike cells of A-T patients that exhibit sensitivity to a narrow range of DNA-damaging agents, ATR inactivation promoted sensitivity to various types of agents, including those affecting DNA replication (Cliby et al., 1998). Therefore, as previously suggested, it was demonstrated that despite a functional overlap with ATM, ATR was preferentially involved in the response to DNA damage caused during DNA replication. It is now clear that ATM recognizes DSBs by association with the MRN complex, whereas ATR recognizes RPA-coated single-stranded DNA, a byproduct of multiple DNA damage and replication arrest agents (Blackford and Jackson, 2017).

\section{Rad53, a S. cerevisiae protein kinase with a central role in DDR}

During inhibition of DNA replication, the S phase checkpoint promotes an arrest of the cell cycle prior to mitosis. To understand the mechanisms regulating this check- 
point, Stephen Elledge's laboratory developed a screen to identify yeast mutants that were sensitive to HU. As mentioned before, mutants isolated in this screen were referred to as sad (S-phase arrest-defective) and included sad1 to sad5. (Allen et al., 1994). Among the isolated mutants, sadl had the highest sensitivity to HU and therefore was selected for further investigation. In addition to presenting dysfunctional S phase checkpoint, sadl mutants had defects in the G1 and G2/M checkpoints (Allen et al., 1994). Interestingly, inhibition of $\mathrm{Cks} 1$, a regulatory subunit of cyclin-dependent kinase Cdc28, rescued sad1 sensitivity to HU (Allen et al., 1994). Considering that Cks1 activity was required for both $\mathrm{G} 1 / \mathrm{S}$ and $\mathrm{G} 2 / \mathrm{M}$ transitions, it was suggested that in response to DNA damage, Sad1 would negatively regulate $\mathrm{Cks} 1$ to prevent its cell cycle transitionpromoting activity. Furthermore, sadl mutants showed a reduction in Dun1 phosphorylation levels associated with a decrease in the expression of RNR2 and RNR3 (Allen et al., 1994). Interestingly, as mentioned in previous sections of this review, while it was demonstrated that Dun1 was required for the transcriptional response to DNA damage, it was not required to control cell cycle checkpoints (Zhou and Elledge, 1993). Considering that sad1 mutants were defective for both cell cycle arrest and RNR expression, it was suggested that Sad1 functions upstream of Dun1 in the signaling pathway responsible for the DNA damage transcriptional response (Figure 2). SAD1 was cloned by complementation assays and its coding region was shown to be identical to $S P K 1$, a previously isolated gene encoding a serine/threonine protein kinase (Stern et al., 1991) (Figure 1). sad1 was also shown to be allelic to rad53, a radiation-sensitive mutant identified in 1974 (Game and Mortimer, 1974). Eventually it was shown that both mutants had the same defective gene and therefore $S A D 1$ was referred to as RAD53 (Allen et al., 1994).

In order to identify genes involved in the regulation of Rad53, Sanchez et al. (1996) performed a screen to identify mutants whose viability depended on the overexpression of Rad53. Surprisingly, one of the isolated mutants was mecl (Kato and Ogawa, 1994; Weinert et al., 1994) (Figure 1). Supporting the functional dependency between Mec1 and Rad53, Mec1 was also shown to be involved in the regulation of the S phase checkpoint (Allen et al., 1994; Weinert et al., 1994; Paulovich and Hartwell, 1995). In addition to salvaging the lethality of a mecl null mutant, the overexpression of RAD53 rescued the HU and UV sensitivity of a mecl partial mutant (Sanchez et al., 1996). Together, these observations suggested that Rad53 mediated the essential function of Mecl and therefore, acted downstream of Mec1 in the DDR pathway (Figure 2). Using a phosphodependent electrophoretic mobility shift assay the authors showed that Rad53 was phosphorylated in response to HU and methyl methanesulfonate (MMS) treatment, demonstrating that its function was actively modulated during DNA replication arrest and DNA damage. The authors also showed that Rad53 phosphorylation decreased in mecl de- fective mutants. Interestingly, an extra copy of TEL1 partially complemented Rad53 phosphorylation levels, reinforcing the idea of a functional overlap between Mec1 and Tel1 (Sanchez et al., 1996) (Figure 2).

The identification and cloning of RAD53 helped to integrate the functions of Mec1 and Tel1 in the regulation of cell cycle checkpoints and dNTPs synthesis (Figure 2). At that point, Mec1, Tel1, Rad53 and Dun1 constituted the central components of a signaling pathway whose phosphorylation cascade would ultimately regulate the DDR (Figure 2). It is important to note that, unlike Dun1, whose transcriptional function in inducing RNR activity is substituted by the transactional factor p53 in human cells (Kastan et al., 1992), Mec1 and Tel1 are orthologs of ATR and ATM, respectively (Table 1 and Figure 2).

\section{Chk1 and Cds1 kinases of $S$. pombe: dual regulators of the DDR}

In addition to studies in $S$. cerevisiae, studies in $S$. pombe had an important contribution in the identification and functional characterization of DDR kinases. In the early 1990's it was known that cell cycle transition to mitosis depended largely on $c d c 2$ (cell division cycle 2 ). To better understand the mechanisms involved in the regulation of Cdc2, Walworth et al. (1993) introduced a multicopy gene library into a temperature-sensitive $c d c 2$ mutant and isolated plasmids that allowed the cells to grow at restrictive temperatures. One of these plasmids carried a gene encoding a serine/threonine kinase referred to as chkl (checkpoint kinase 1) (Figure 1). Almost at the same time, al-Khodaire et al. (1994) isolated a gene that complemented the checkpoint defects of a rad 27 (radiation sensitive 27) mutant. This gene was found to be identical to the chkl gene previously isolated by Walworth et al. (1993) and, by convention, it was referred to by the same name.

Supporting the role of Chk1 in cell cycle arrest, chkl null mutants presented mitotic checkpoint defects and increased sensitivity in response to UV and IR treatments (Walworth et al., 1993; al-Khodairy et al., 1994). Chk1 was phosphorylated in response to UV, MMS and IR treatments, suggesting that the kinase was actively regulated during DDR. In addition, it was demonstrated that a kinase-dead mutant of Chk1 was more sensitive to UV than the wild type, implying that its kinase activity was also important for cellular response to DNA damage. Further experiments showed a reduction on Chk1 phosphorylation in several rad mutants, including rad3 (Walworth and Bernards, 1996). These observations suggested that in S. pombe, $\operatorname{Rad} 3$ acted upstream to Chk1 in the regulation of mitotic checkpoint (Figure 2). Interestingly, chkl null mutants displayed hypersensitivity and mitotic checkpoint defects when treated with UV, MMS and IR but not with HU. Moreover, Chk1 did not undergo changes in phosphorylation levels in HU-treated cells (Walworth and Bernards, 1996). 
In 1995, the serine/threonine kinase Cds1 (checking DNA synthesis 1 ) was identified as a suppressor of a temperature-sensitive mutant of polymerase $\alpha$ (Murakami and Okayama, 1995) (Figure 1). cds1 null mutants showed loss of viability associated with S phase entry in the presence of HU. Supporting its role during DNA replication, Cds1 was phosphorylated and activated in response to HU. Additionally, $c d s 1$ null mutants lost the ability to slow $\mathrm{S}$ phase in HU-treated cells (Lindsay et al., 1998). This observation suggested that while Chk1 responded to DNA damage, Cds1 preferentially responded to DNA replication arrest. However, in $c d s 1$ null mutants Chk1 could be activated in response to HU treatment, suggesting that this dynamic was not so simplistic. In fact, $\operatorname{chk} 1 \mathrm{cds} 1$ double mutants were more sensitive to HU than single mutants alone, suggesting some functional overlap between the two kinases (Boddy et al., 1998; Lindsay et al., 1998). Corroborating these observations, Paul Russell's laboratory showed that both Chk1 and $\mathrm{Cds} 1$ regulate $\mathrm{Cdc} 2$, the kinase responsible for mitosis initiation. While Chk1 and Cds1 inhibit Cdc25 indirectly by repressing $\mathrm{Cdc} 2, \mathrm{Cds} 1$ was required to increase the abundance of Mik1, a Cdc2 repressor (Furnari et al., 1997; Rhind et al., 1997; Boddy et al., 1998; Baber-Furnari et al., 2000; Christensen et al., 2000). Thus, although Cds1 and Chk1 regulate sub pathways of $\mathrm{Rad} 3$ response, both $\mathrm{Cds} 1$ and Chk1 (Figure 2) are able to control the mitotic checkpoint in response to DNA replication arrest.

Despite the structural similarity, Cds1 and Rad53 show marked differences in their functions throughout the cell cycle (Figure 2). While Rad53 has a broader role, acting along G1, S and G2 phases, Cds1 acts only during $\mathrm{S}$ phase (Figure 2). In this context, researchers have directed their attention to the existence of a potential mammalian counterpart of Rad53 and Chk1, which could aid understanding of how mammalian cells transduce their signals to regulate DDR.

\section{CHK1 and CHK2: linking DNA damage to cell cycle checkpoints in mammalian cells}

To identify the human ortholog of $S$. cerevisiae RAD53 and S. pombe cds1, Matsuoka et al. (1998) used the information of an EST with sequence similarity to the conserved FHA domain of Rad53 and Cds1 to screen a human cDNA library. By using this strategy, the authors isolated the cDNA encoding a protein with $26 \%$ identity with both Rad53 and Cds1. This gene was name CHK2 (checkpoint kinase) in reference to $C H K 1$, a gene of similar function identified a few months earlier by the same research group (Sanchez et al., 1997) (Figure 1). Supporting functional conservation with RAD53, the expression of $C H K 2$ complemented the lethality of rad53 null mutants. In addition, as demonstrated for Rad53, cells exposed to UV or IR showed an increase in CHK2 phosphorylation levels suggesting that its function was actively modulated during DDR (Matsuoka et al., 1998). At that time, the phosphatase
CDC25 was already known for its role in regulating cell cycle progression (Walworth et al., 1993; Furnari et al., 1999). Considering the role of Rad53 in cell cycle checkpoints regulation, the authors demonstrated that $\mathrm{CHK} 2$ phosphorylated CDC25A, CDC25B and CDC25C. Using a kinase-dead allele from $C H K 2$, the authors mapped a phosphorylation site at serine 216 of CDC25C, a site known to be involved in its negative regulation (Ogg et al., 1994). Moreover, the kinase activity of CHK2 was shown to be dependent on treatment with UV, IR or HU suggesting that CHK2-dependent phosphorylation of CDC25C is modulated in response to DNA damage (Matsuoka et al., 1998).

To test whether CHK2 function depended on signals elicited by upstream kinases, the authors evaluated the phosphorylation status and activity of CHK2 on A-T irradiated cells. The experiment indicated a reduction in $\mathrm{CHK} 2$ phosphorylation associated with a decrease in its kinase activity. Finally, the ectopic expression of ATM rescued both phosphorylation status and activity of CHK2, suggesting that ATM is located upstream to CHK2 in a signaling pathway responsible for regulating the cell cycle checkpoints (Matsuoka et al., 1998) (Figure 2).

A few months before the identification of CHK2, Sanchez et al. (1997) used a degenerate PCR strategy and identified $C H K 1$, a human gene very similar to $S$. pombe chk1 (Table 1 and Figure 1). In parallel, CHK1 was also isolated in Tony Carr's laboratory (Flaggs et al., 1997). Human CHK1 showed an increase in DNA damage-dependent phosphorylation, suggesting that like its yeast counterpart (Walworth and Bernards, 1996), its function was modulated during DDR. In addition, as observed for CHK2, CHK1 phosphorylated CDC25C at serine 216 (Sanchez et al., 1997; Matsuoka et al., 1998). This result demonstrated the existence of two DNA damage responsive kinases capable of promoting the same inhibitory signal in CDC25C. Despite the functional redundancy, it was suggested that CHK1 and CHK2 could play different roles depending on the type of damage elicited and/or the stage of the cell cycle in which they were active (Figure 2). It was also possible that they were specific for other unknown substrates. Interestingly, unlike CHK2 whose regulation was attributed to ATM-mediated signaling, which PIKK was responsible for regulating CHK1 in mammalian cells remained unknown. Eventually, through the use of conditional CHK1-deficient cell lines and a dominant negative mutant of ATR, it was demonstrated that the regulation of CHK1 was indeed dependent on ATR (Liu et al., 2000) (Figure 2).

\section{S. cerevisiae Chk1, a DDR kinase involved in mitotic arrest}

Based on the $S$. pombe and human sequences of $C H K 1$, the $S$. cerevisiae ortholog was identified by similarity with the unknown ORF YBR274w (Figure 1). Unlike its human counterpart, $S$. cerevisiae chk1 mutants were not essential to cell viability. Despite no evident alteration in the 
S phase checkpoint, chk1 mutants synchronized with nocodazole, an inhibitor of microtubule polymerization, showed moderate sensitivity and defects in cell cycle arrest in response to IR treatment, suggesting a potential involvement of Chkl in the regulation of mitosis progression (Sanchez et al., 1999). Chk1 was phosphorylated in response to DNA damage. Moreover, phosphorylation of both Chk1 and Rad53 was shown to be dependent on Mec1 but independent from each other (Sanchez et al., 1999). These results indicated that both Rad53 and Chk1 were independently regulated by Mec1 (Figure 2). Further experiments showed that different from its $S$. pombe and human ortholog, the $S$. cerevisiae Chk1 promoted cell cycle arrest through a different mechanism involving the regulation of Pds1. Pds1, known as a Securin, prevents the segregation of sister chromatids thus inhibiting anaphase entry. By combining yeast genetics and biochemical approaches, Sanchez et al demonstrated that during DDR, the CHK1-mediated response promotes the stability of Pds1, thus contributing to cell cycle arrest prior to anaphase entry (Sanchez et al., 1999).

The identification and functional characterization of S. cerevisiae Chk1 showed that, unlike Rad53, which promotes cell cycle arrest during G1/S, S phase and G2/M transitions, Chk1 promotes cell cycle arrest during mitosis. These observations suggested that Rad53 and Chk1 could be acting in parallel to reinforce DDR through a fail-safe mechanism that guarantees cell cycle arrest at different stages (Figure 2).

\section{A brief consideration on DNA-PK, a DDR kinase with no homologues in yeast}

In 1986, the group of Carl W. Anderson accidentally discovered that linear fragments of dsDNA induced the phosphorylation of several proteins in extracts of widely divergent metazoan species (Walker et al., 1985). In the following years, several laboratories identified the protein responsible for this kinase activity as DNA-PKcs (DNAdependent protein kinase catalytic subunit), another member of the PIKK family. It was latter established that DNA$\mathrm{PK}$ is recruited to DSBs by the heterodimeric Ku complex to promote DSB repair by non-homologous end joining (NHEJ). Therefore, in addition to ATM and ATR, mammalian DDR is also coordinated by DNA-PKcs (Blackford and Jackson, 2017). It is important to note that although yeast presents all core NHEJ factors, it lacks the catalytic DNA-PKcs. In this case, other factors such as Mre11, Rad50 and Xrs2 (MRX complex), may compensate for the lack of DNA-PK.

\section{DDR kinases in the $21^{\text {st }}$ century: advances and perspectives}

Since the discovery of the DDR kinases, the identification of their substrates progressed slowly with only a few targets being identified during the late 1990s and early 2000s. However, over the last decade, technical advances in phosphoproteomics had a profound impact in the DDR field, expanding the identification of proteins phosphorylated by the DDR kinases in both yeast and human cells (Beausoleil et al., 2004; Olsen et al., 2006; Albuquerque et al., 2008). Furthermore, the advent of quantitative mass spectrometry analysis allowed researchers to monitor the dynamics of DNA damage signaling by looking simultaneously at multiple DDR kinase substrates in a systematic and unbiased manner (Bastos de Oliveira et al., 2015; Willis et al., 2016; Zhou et al., 2016; Lanz et al., 2018; Bass and Cortez, 2019). For instance, a recent study based on quantitative phosphoproteomics showed that the human DDR activators ETAA1 and TopBP1 regulate distinct aspects of ATR signaling. By monitoring ATR-dependent phosphorylation events in ETAA1 and/or TopBP1 deficient cells, the authors revealed that while TopBP1 is the primary ATR activator of replication stress, ETAA1 coordinates ATR signaling during mitosis (Bass and Cortez, 2019).

Recently, the advent of genome-editing tools in human cell lines, combined with new DDR kinase inhibitors, have been successfully applied for the screen of synthetic lethal interactions, offering new insights for cancer treatment (Ruiz et al., 2016; Gerhards and Rottenberg, 2018; Wang et al., 2019). The kinase ATR, for example, have come under the spotlight in recent years as prominent therapeutic target in cancer (Foote et al., 2015). In order to cope with high levels of replication stress, cancer cells depend on ATR for survival and proliferation (Choi et al., 2011; Toledo et al., 2011a). Therefore, pharmacological inhibition of ATR selectively sensitizes different types of tumor cells, especially in tumors with defects in the ATM-p53 pathway (Charrier et al., 2011; Peasland et al., 2011; Reaper et al., 2011; Toledo et al., 2011b; Fokas et al., 2012; Foote et al., 2013, 2015,2018; Kim et al., 2018).

The knowledge accumulated over the last three decades, since the discovery of the first DDR kinase in yeast, was fundamental to our understanding of how cells coordinate the multiple responses that confer protection against genomic instability (Ciccia and Elledge, 2010). Moreover, besides their importance for genome integrity, it has been suggested that DDR kinases regulate other biological processes such as protein homeostasis, carbon and phosphatidylinositol metabolism, vesicle trafficking, and autophagy (Simpson-Lavy et al., 2015; Zhou et al., 2016; Dahl and Aird, 2017; Yi et al., 2017; Cheng et al., 2018; CorcolesSaez et al., 2018, 2019). However, it remains to be established the relevance of these processes during DDR. Finally, the recent discovery that DDR kinases are activated by oncogenic stress (Halazonetis et al., 2008) put them as promising targets for clinical applications. Thus, whether for the basic or translational research aspect, there is plenty of space to continue exploring the biology of DDR kinases in both yeast and humans. 


\section{Acknowledgments}

This work was supported by grants from Fundação de Amparo à Pesquisa do Estado de São Paulo (FAPESP 2018/05417-0) to JRRC and Fundação Carlos Chagas Filho de Amparo à Pesquisa do Estado do Rio de Janeiro (FAPERJ E-26/010.002366/2016) to FMBdO. BLS was supported by a fellowship from Conselho Nacional de Desenvolvimento Científico e Tecnológico (CNPq)

\section{Conflict of Interest}

The authors declare that there is no conflict of interest that could be perceived as prejudicial to the impartiality of the reported research.

\section{Authors Contributions}

JRRC, BLS and FMBdO wrote the manuscript; all authors read and approved the final version.

\section{References}

al-Khodairy F and Carr AM (1992) DNA repair mutants defining G2 checkpoint pathways in Schizosaccharomyces pombe. EMBO J 11:1343-1350.

al-Khodairy F, Fotou E, Sheldrick KS, Griffiths DJ, Lehmann AR and Carr AM (1994) Identification and characterization of new elements involved in checkpoint and feedback controls in fission yeast. Mol Biol Cell 5:147-160.

Albuquerque CP, Smolka MB, Payne SH, Bafna V, Eng J and Zhou H (2008) A multidimensional chromatography technology for in-depth phosphoproteome analysis. Mol Cell Proteomics 7:1389-1396.

Allen JB, Zhou Z, Siede W, Friedberg EC and Elledge SJ (1994) The SAD1/RAD53 protein kinase controls multiple checkpoints and DNA damage-induced transcription in yeast. Genes Dev 8:2401-2415.

Baber-Furnari BA, Rhind N, Boddy MN, Shanahan P, LopezGirona A and Russell P (2000) Regulation of mitotic inhibitor Mik1 helps to enforce the DNA damage checkpoint. Mol Biol Cell 11:1-11.

Bass TE and Cortez D (2019) Quantitative phosphoproteomics reveals mitotic function of the ATR activator ETAA1. J Cell Biol 218:1235-1249.

Bastos de Oliveira FM, Kim D, Cussiol JR, Das J, Jeong MC, Doerfler L, Schmidt KH, Yu H and Smolka MB (2015) Phosphoproteomics reveals distinct modes of Mec1/ATR signaling during DNA replication. Mol Cell 57:1124-1132.

Beamish H, Khanna KK and Lavin MF (1994) Ionizing radiation and cell cycle progression in ataxia telangiectasia. Radiat Res 138:S130-133.

Beausoleil SA, Jedrychowski M, Schwartz D, Elias JE, Villen J, Li J, Cohn MA, Cantley LC and Gygi SP (2004) Large-scale characterization of HeLa cell nuclear phosphoproteins. Proc Natl Acad Sci USA 101:12130-12135.

Bentley NJ, Holtzman DA, Flaggs G, Keegan KS, DeMaggio A, Ford JC, Hoekstra M and Carr AM (1996) The Schizosaccharomyces pombe rad3 checkpoint gene. EMBO J 15:6641-6651.
Blackford AN and Jackson SP (2017) ATM, ATR, and DNA-PK: The trinity at the heart of the DNA damage response. Mol Cell 66:801-817.

Boddy MN, Furnari B, Mondesert O and Russell P (1998) Replication checkpoint enforced by kinases Cds1 and Chk1. Science 280:909-912.

Charrier JD, Durrant SJ, Golec JM, Kay DP, Knegtel RM, MacCormick S, Mortimore M, O'Donnell ME, Pinder JL, Reaper PM et al. (2011) Discovery of potent and selective inhibitors of ataxia telangiectasia mutated and Rad3 related (ATR) protein kinase as potential anticancer agents. J Med Chem 54:320-2330.

Cheng A, Zhao T, Tse KH, Chow HM, Cui Y, Jiang L, Du S, Loy MMT and Herrup K (2018) ATM and ATR play complementary roles in the behavior of excitatory and inhibitory vesicle populations. Proc Natl Acad Sci U S A 115:E292E301.

Choi S, Toledo LI, Fernandez-Capetillo O and Bakkenist CJ (2011) CGK733 does not inhibit ATM or ATR kinase activity in H460 human lung cancer cells. DNA Repair 10:10001001.

Christensen PU, Bentley NJ, Martinho RG, Nielsen O and Carr AM (2000) Mik1 levels accumulate in S phase and may mediate an intrinsic link between $\mathrm{S}$ phase and mitosis. Proc Natl Acad Sci U S A 97:2579-2584.

Ciccia A and Elledge SJ (2010) The DNA damage response: making it safe to play with knives. Mol Cell 40:179-204.

Cimprich KA, Shin TB, Keith CT and Schreiber SL (1996) cDNA cloning and gene mapping of a candidate human cell cycle checkpoint protein. Proc Natl Acad Sci U S A 93:28502855.

Cliby WA, Roberts CJ, Cimprich KA, Stringer CM, Lamb JR, Schreiber SL and Friend SH (1998) Overexpression of a kinase-inactive ATR protein causes sensitivity to DNAdamaging agents and defects in cell cycle checkpoints. EMBO J 17:159-169.

Corcoles-Saez I, Dong K, Johnson AL, Waskiewicz E, Costanzo M, Boone C and Cha RS (2018) Essential function of Mec1, the Budding Yeast ATM/ATR Checkpoint-Response Kinase, in protein homeostasis. Dev Cell 46:495-503.e2.

Corcoles-Saez I, Dong K and Cha RS (2019) Versatility of the Mec1(ATM/ATR) signaling network in mediating resistance to replication, genotoxic, and proteotoxic stresses. Curr Genet 65:657-661.

Dahl ES and Aird KM (2017) Ataxia-telangiectasia mutated modulation of carbon metabolism in cancer. Front Oncol 7:291.

Elledge SJ (2015) Accidents and damage control. Cell 162:11961200.

Elledge SJ and Davis RW (1987) Identification and isolation of the gene encoding the small subunit of ribonucleotide reductase from Saccharomyces cerevisiae: DNA damage-inducible gene required for mitotic viability. Mol Cell Biol 7:2783-2793.

Elledge SJ and Davis RW (1989) DNA damage induction of ribonucleotide reductase. Mol Cell Biol 9:4932-4940.

Elledge SJ and Davis RW (1990) Two genes differentially regulated in the cell cycle and by DNA-damaging agents encode alternative regulatory subunits of ribonucleotide reductase. Genes Dev 4:740-751. 
Falck J, Coates J and Jackson SP (2005) Conserved modes of recruitment of ATM, ATR and DNA-PKcs to sites of DNA damage. Nature 434:605-611.

Flaggs G, Plug AW, Dunks KM, Mundt KE, Ford JC, Quiggle MR, Taylor EM, Westphal CH, Ashley T, Hoekstra MF et al. (1997) Atm-dependent interactions of a mammalian chk1 homolog with meiotic chromosomes. Curr Biol 7:977-986.

Fokas E, Prevo R, Pollard JR, Reaper PM, Charlton PA, Cornelissen B, Vallis KA, Hammond EM, Olcina MM, Gillies McKenna $\mathrm{W}$ et al. (2012) Targeting ATR in vivo using the novel inhibitor VE-822 results in selective sensitization of pancreatic tumors to radiation. Cell Death Dis 3:e441.

Foote KM, Blades K, Cronin A, Fillery S, Guichard SS, Hassall L, Hickson I, Jacq X, Jewsbury PJ, McGuire TM et al. (2013) Discovery of 4-\{4-[(3R)-3-Methylmorpholin-4-yl]-6-[1(methylsulfonyl)cyclopropyl]pyrimidin-2-y 1$\}-1 \mathrm{H}$-indole (AZ20): A potent and selective inhibitor of ATR protein kinase with monotherapy in vivo antitumor activity. J Med Chem 56:2125-2138.

Foote KM, Lau A and Nissink JW (2015) Drugging ATR: Progress in the development of specific inhibitors for the treatment of cancer. Future Med Chem 7:873-891.

Foote KM, Nissink JWM, McGuire T, Turner P, Guichard S, Yates JWT, Lau A, Blades K, Heathcote D, Odedra R et al. (2018) Discovery and characterization of AZD6738, a potent inhibitor of Ataxia Telangiectasia mutated and Rad3 related (ATR) kinase with application as an anticancer agent. J Med Chem 61:9889-9907.

Friedberg EC (2008) A brief history of the DNA repair field. Cell Res 18:3-7.

Furnari B, Rhind N and Russell P (1997) Cdc25 mitotic inducer targeted by chk1 DNA damage checkpoint kinase. Science 277:1495-1497.

Furnari B, Blasina A, Boddy MN, McGowan CH and Russell P (1999) Cdc25 inhibited in vivo and in vitro by checkpoint kinases Cds1 and Chk1. Mol Biol Cell 10:833-845.

Game JC and Mortimer RK (1974) A genetic study of x-ray sensitive mutants in yeast. Mutat Res 24:281-292.

Gatti RA, Berkel I, Boder E, Braedt G, Charmley P, Concannon P, Ersoy F, Foroud T, Jaspers NG, Lange K et al. (1988) Localization of an ataxia-telangiectasia gene to chromosome 11q22-23. Nature 336:577-580.

Gerhards NM and Rottenberg S (2018) New tools for old drugs: Functional genetic screens to optimize current chemotherapy. Drug Resist Updat 36:30-46.

Giglia-Mari G, Zotter A and Vermeulen W (2011) DNA damage response. Cold Spring Harb Perspect Biol 3:a000745.

Greenwell PW, Kronmal SL, Porter SE, Gassenhuber J, Obermaier B and Petes TD (1995) TEL1, a gene involved in controlling telomere length in S. cerevisiae, is homologous to the human ataxia telangiectasia gene. Cell 82:823-829.

Halazonetis TD, Gorgoulis VG and Bartek J (2008) An oncogene-induced DNA damage model for cancer development. Science 319:1352-1355.

Jimenez G, Yucel J, Rowley R and Subramani S (1992) The rad3+ gene of Schizosaccharomyces pombe is involved in multiple checkpoint functions and in DNA repair. Proc Natl Acad Sci USA 89:4952-4956.

Kastan MB, Onyekwere O, Sidransky D, Vogelstein B and Craig RW (1991) Participation of p53 protein in the cellular response to DNA damage. Cancer Res 51:6304-6311.
Kastan MB, Zhan Q, el-Deiry WS, Carrier F, Jacks T, Walsh WV, Plunkett BS, Vogelstein B and Fornace Jr AJ (1992) A mammalian cell cycle checkpoint pathway utilizing p53 and GADD45 is defective in ataxia-telangiectasia. Cell 71:587-597.

Kato $\mathrm{R}$ and Ogawa $\mathrm{H}$ (1994) An essential gene, ESR1, is required for mitotic cell growth, DNA repair and meiotic recombination in Saccharomyces cerevisiae. Nucleic Acids Res 22:3104-3112.

Keith CT and Schreiber SL (1995) PIK-related kinases: DNA repair, recombination, and cell cycle checkpoints. Science 270:50-51.

Kim D, Liu Y, Oberly S, Freire R and Smolka MB (2018) ATRmediated proteome remodeling is a major determinant of homologous recombination capacity in cancer cells. Nucleic Acids Res 46:8311-8325.

Lambert S and Carr AM (2013) Replication stress and genome rearrangements: lessons from yeast models. Curr Opin Genet Dev 23:132-139.

Lange E, Borresen AL, Chen X, Chessa L, Chiplunkar S, Concannon P, Dandekar S, Gerken S, Lange K, Liang T et al. (1995) Localization of an ataxia-telangiectasia gene to an approximately $500-\mathrm{kb}$ interval on chromosome 11q23.1: Linkage analysis of 176 families by an international consortium. Am J Hum Genet 57:112-119.

Lanz MC, Oberly S, Sanford EJ, Sharma S, Chabes A and Smolka MB (2018) Separable roles for Mec1/ATR in genome maintenance, DNA replication, and checkpoint signaling. Genes Dev 32:822-835.

Lindahl T (1993) Instability and decay of the primary structure of DNA. Nature 362:709-715.

Lindahl T and Nyberg B (1972) Rate of depurination of native deoxyribonucleic acid. Biochemistry 11:3610-3618.

Lindsay HD, Griffiths DJ, Edwards RJ, Christensen PU, Murray JM, Osman F, Walworth N and Carr AM (1998) S-phasespecific activation of Cds1 kinase defines a subpathway of the checkpoint response in Schizosaccharomyces pombe. Genes Dev 12:382-395.

Liu Q, Guntuku S, Cui XS, Matsuoka S, Cortez D, Tamai K, Luo G, Carattini-Rivera S, DeMayo F, Bradley A et al. (2000) Chk1 is an essential kinase that is regulated by Atr and required for the G(2)/M DNA damage checkpoint. Genes Dev 14:1448-1459.

Lowe SW, Schmitt EM, Smith SW, Osborne BA and Jacks T (1993) p53 is required for radiation-induced apoptosis in mouse thymocytes. Nature 362:847-849.

Marechal A and Zou L (2013) DNA damage sensing by the ATM and ATR kinases. Cold Spring Harb Perspect Biol 5:a012716.

Matsuoka S, Huang M and Elledge SJ (1998) Linkage of ATM to cell cycle regulation by the Chk2 protein kinase. Science 282:1893-1897.

McConville CM, Byrd PJ, Ambrose HJ and Taylor AM (1994) Genetic and physical mapping of the ataxia-telangiectasia locus on chromosome 11q22-q23. Int J Radiat Biol 66:S45S56.

Morrow DM, Tagle DA, Shiloh Y, Collins FS and Hieter P (1995) TEL1, a S. cerevisiae homolog of the human gene mutated in ataxia telangiectasia, is functionally related to the yeast checkpoint gene MEC1. Cell 82:831-840. 
Murakami H and Okayama H (1995) A kinase from fission yeast responsible for blocking mitosis in $\mathrm{S}$ phase. Nature 374:817-819.

Naito T, Matsuura A and Ishikawa F (1998) Circular chromosome formation in a fission yeast mutant defective in two ATM homologues. Nat Genet 20:203-206.

Ogg S, Gabrielli B and Piwnica-Worms H (1994) Purification of a serine kinase that associates with and phosphorylates human Cdc25C on serine 216. J Biol Chem 269:30461-30469.

Olsen JV, Blagoev B, Gnad F, Macek B, Kumar C, Mortensen P and Mann M (2006) Global, in vivo, and site-specific phosphorylation dynamics in signaling networks. Cell 127:635648.

Paulovich AG and Hartwell LH (1995) A checkpoint regulates the rate of progression through $\mathrm{S}$ phase in $S$. cerevisiae in response to DNA damage. Cell 82:841-847.

Peasland A, Wang LZ, Rowling E, Kyle S, Chen T, Hopkins A, Cliby WA, Sarkaria J, Beale G, Edmondson RJ et al. (2011) Identification and evaluation of a potent novel ATR inhibitor, NU6027, in breast and ovarian cancer cell lines. Br J Cancer 105:372-381.

Reaper PM, Griffiths MR, Long JM, Charrier JD, Maccormick S, Charlton PA, Golec J and Pollard JR (2011) Selective killing of ATM- or p53-deficient cancer cells through inhibition of ATR. Nat Chem Biol 7:428-430.

Rhind N, Furnari B and Russell P (1997) Cdc2 tyrosine phosphorylation is required for the DNA damage checkpoint in fission yeast. Genes Dev 11:504-511.

Rotman G, Savitski K, Vanagaite L, Bar-Shira A, Ziv Y, Gilad S, Uchenik V, Smith S and Shiloh Y (1994) Physical and genetic mapping at the ATA/ATC locus on chromosome 11q22-23. Int J Radiat Biol 66:S63-S66.

Ruiz S, Mayor-Ruiz C, Lafarga V, Murga M, Vega-Sendino M, Ortega $\mathrm{S}$ and Fernandez-Capetillo O (2016) A genome-wide CRISPR screen identifies CDC25A as a determinant of sensitivity to ATR inhibitors. Mol Cell 62:307-313.

Sanchez Y, Desany BA, Jones WJ, Liu Q, Wang B and Elledge SJ (1996) Regulation of RAD53 by the ATM-like kinases MEC1 and TEL1 in yeast cell cycle checkpoint pathways. Science 271:357-360.

Sanchez Y, Wong C, Thoma RS, Richman R, Wu Z, PiwnicaWorms H and Elledge SJ (1997) Conservation of the Chk1 checkpoint pathway in mammals: Linkage of DNA damage to Cdk regulation through Cdc25. Science 277:1497-1501.

Sanchez Y, Bachant J, Wang H, Hu F, Liu D, Tetzlaff M and Elledge SJ (1999) Control of the DNA damage checkpoint by chk1 and rad53 protein kinases through distinct mechanisms. Science 286:1166-1171.

Savitsky K, Bar-Shira A, Gilad S, Rotman G, Ziv Y, Vanagaite L, Tagle DA, Smith S, Uziel T, Sfez S et al. (1995a) A single ataxia telangiectasia gene with a product similar to PI-3 kinase. Science 268:1749-1753.

Savitsky K, Sfez S, Tagle DA, Ziv Y, Sartiel A, Collins FS, Shiloh Y and Rotman G (1995b) The complete sequence of the coding region of the $A T M$ gene reveals similarity to cell cycle regulators in different species. Hum Mol Genet 4:20252032.

Shiloh Y and Rotman G (1996) Ataxia-telangiectasia and the ATM gene: Linking neurodegeneration, immunodeficiency, and cancer to cell cycle checkpoints. J Clin Immunol 16:254-260.

Simpson-Lavy KJ, Bronstein A, Kupiec M and Johnston M (2015) Cross-talk between carbon metabolism and the DNA damage response in S. cerevisiae. Cell Rep 12:1865-1875.

Stern DF, Zheng P, Beidler DR and Zerillo C (1991) Spk1, a new kinase from Saccharomyces cerevisiae, phosphorylates proteins on serine, threonine, and tyrosine. Mol Cell Biol 11:987-1001.

Toledo LI, Murga M and Fernandez-Capetillo O (2011a) Targeting ATR and Chk1 kinases for cancer treatment: A new model for new (and old) drugs. Mol Oncol 5:368-373.

Toledo LI, Murga M, Zur R, Soria R, Rodriguez A, Martinez S, Oyarzabal J, Pastor J, Bischoff JR and Fernandez-Capetillo O (2011b) A cell-based screen identifies ATR inhibitors with synthetic lethal properties for cancer-associated mutations. Nat Struct Mol Biol 18:721-727.

Walker AI, Hunt T, Jackson RJ and Anderson CW (1985) Double-stranded DNA induces the phosphorylation of several proteins including the $90000 \mathrm{~mol}$. wt. heat-shock protein in animal cell extracts. EMBO J 4:139-145.

Walworth NC and Bernards R (1996) rad-dependent response of the chk1-encoded protein kinase at the DNA damage checkpoint. Science 271:353-356.

Walworth N, Davey S and Beach D (1993) Fission yeast chk1 protein kinase links the rad checkpoint pathway to cdc2. Nature 363:368-371.

Wang C, Wang G, Feng X, Shepherd P, Zhang J, Tang M, Chen Z, Srivastava M, McLaughlin ME, Navone NM et al. (2019) Genome-wide CRISPR screens reveal synthetic lethality of RNASEH2 deficiency and ATR inhibition. Oncogene 38:2451-2463.

Weinert TA and Hartwell LH (1993) Cell cycle arrest of cdc mutants and specificity of the RAD9 checkpoint. Genetics 134:63-80

Weinert TA, Kiser GL and Hartwell LH (1994) Mitotic checkpoint genes in budding yeast and the dependence of mitosis on DNA replication and repair. Genes Dev 8:652-665.

Willis NA, Zhou C, Elia AE, Murray JM, Carr AM, Elledge SJ and Rhind N (2016) Identificatio of S-phase DNA damage-response targets in fission yeast reveals conservation of damage-response networks. Proc Natl Acad Sci U S A 113:E3676-E3685.

Yi C, Tong J, Lu P, Wang Y, Zhang J, Sun C, Yuan K, Xue R, Zou B, Li N et al. (2017) Formation of a Snf1-Mec1-Atg1 module on mitochondria governs energy deprivation-induced autophagy by regulating mitochondrial respiration. Dev Cell 41:59-71.e4.

Zhou C, Elia AE, Naylor ML, Dephoure N, Ballif BA, Goel G, Xu Q, Ng A, Chou DM, Xavier RJ et al. (2016) Profiling DNA damage-induced phosphorylation in budding yeast reveals diverse signaling networks. Proc Natl Acad Sci U S A 113:E3667-E3675.

Zhou Z and Elledge SJ (1993) DUN1 encodes a protein kinase that controls the DNA damage response in yeast. Cell 75:1119-1127.

\section{Associate Editor: Luis Mariano Polo}

License information: This is an open-access article distributed under the terms of the Creative Commons Attribution License (type CC-BY), which permits unrestricted use distribution and reproduction in any medium, provided the original article is properly cited. 